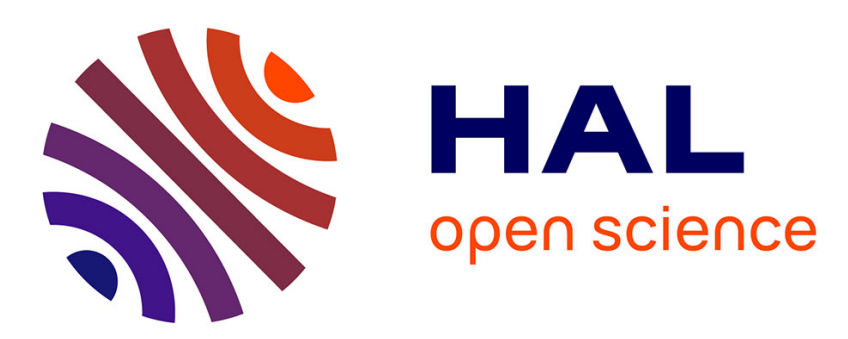

\title{
On self-dual and LCD quasi-twisted codes of index two over a special chain ring
}

\author{
Minjia Shi, Liqin Qian, Patrick Sole
}

\section{To cite this version:}

Minjia Shi, Liqin Qian, Patrick Sole. On self-dual and LCD quasi-twisted codes of index two over a special chain ring. Cryptography and Communications - Discrete Structures, Boolean Functions and Sequences , 2019, 11, pp.717 - 734. 10.1007/s12095-018-0322-5 . hal-02411619

\section{HAL Id: hal-02411619 \\ https://hal.science/hal-02411619}

Submitted on 17 Dec 2019

HAL is a multi-disciplinary open access archive for the deposit and dissemination of scientific research documents, whether they are published or not. The documents may come from teaching and research institutions in France or abroad, or from public or private research centers.
L'archive ouverte pluridisciplinaire HAL, est destinée au dépôt et à la diffusion de documents scientifiques de niveau recherche, publiés ou non, émanant des établissements d'enseignement et de recherche français ou étrangers, des laboratoires publics ou privés. 


\title{
On self-dual and LCD quasi-twisted codes of index two over a special chain ring
}

\author{
Liqin Qian ${ }^{2} \cdot$ Minjia Shi ${ }^{1,2} \cdot$ Patrick Solé ${ }^{3}$
}

Received: 26 April 2018 / Accepted: 25 July 2018 / Published online: 13 August 2018

(C) Springer Science+Business Media, LLC, part of Springer Nature 2018

\begin{abstract}
Let $q$ be a prime power, and let $\mathbb{F}_{q}$ denote the finite field of order $q$. Consider the chain ring $R_{k}=\mathbb{F}_{q}[u] /\left\langle u^{k}\right\rangle$ with $k \geq 1$ an integer. We study self-dual and LCD quasi-twisted codes of index two and twisting constant $\lambda$ over $R_{k}$ for the metric induced by the standard Gray map. Some special factorizations of $x^{m}-\lambda$ over $R_{k}$ are studied. By random coding, we obtain four classes of asymptotically good self-dual $\lambda$-circulant codes and four classes of asymptotically good LCD $\lambda$-circulant codes over $R_{k}$.
\end{abstract}

Keywords Double circulant codes - Gray map - Self-dual codes · LCD codes · Quasi-twisted codes

Mathematics Subject Classification (2010) 94 B15 · 94 B25 · 05 E30

This research is supported by National Natural Science Foundation of China (61672036), Excellent Youth Foundation of Natural Science Foundation of Anhui Province (1808085J20), Technology Foundation for Selected Overseas Chinese Scholar, Ministry of Personnel of China (05015133) and Key projects of support program for outstanding young talents in Colleges and Universities (gxyqZD2016008).

Minjia Shi

smjwcl.good@163.com

Liqin Qian

qianliqin_1108@163.com

Patrick Solé

sole@enst.fr

1 Key Laboratory of Intelligent Computing Signal Processing, Ministry of Education, Anhui University, No.3 Feixi Road, Hefei, Anhui, 230039, China

2 School of Mathematical Sciences, Anhui University, Hefei, Anhui, 230601, China

3 CNRS/LAGA, University of Paris 8, 93526 Saint-Denis, France 


\section{Introduction}

Self-dual codes are important for a number of practical and theoretical reasons, as witnessed by $[1,3,12]$. Another important class of codes defined by their duality properties is that of Linear codes with Complementary Duals (LCD), which were introduced by Massey in 1992 for information theoretic reasons (see [18]). They have found applications recently in Boolean masking [6, 7]. Massey [18] shows that LCD codes are asymptotically good. LCD codes are universal: for $q>3$ there is an algorithm that turns any linear code into an equivalent LCD code [5]. Still, it is of interest to find direct methods of construction of LCD codes as in [4]. One such method is to use quasi-cyclic and quasi-twisted codes. In that direction, self-dual double circulant (resp. double negacirculant) codes over finite fields have been studied recently in [1,3], from the viewpoint of enumeration and asymptotic performance. Some classes of quasi-twisted codes have been studied over finite chain rings in [19]. In [2], A. Alahmadi et al. have studied the linear complementary-dual multinegacirculant codes. Motivated by the techniques in $[1-3,8,19$, 20], we use the Chinese Remainder Theorem (CRT) approach to quasi-twisted codes as introduced in $[11,13,14]$. In particular, we study two classes of self-dual (resp. LCD) negacirculant codes of index 2 over $R_{k}$. Combining with [19], we study two families of factorizations of $x^{m}-\lambda$ over $R_{k}$ with $m$ an odd prime, $\operatorname{gcd}(m, q)=1$. When these special factorizations are thus enforced, we derive exact enumeration formulae, and obtain asymptotic lower bounds on the minimum Hamming distance of the Gray image of these codes.

The material is arranged as follows. In Section 2, we give some background materials on the ring $R_{k}$ and study the case when the element -1 is a square in $R_{k}$. In Section 3 , we derive the enumeration formulae of self-dual (resp. LCD) double circulant and double negacirculant codes of co-index $m$ and we study the special factorizations of $x^{m}-\lambda$ with $m$ an odd prime, and $\operatorname{gcd}(m, q)=1$. Then, we also obtain the enumeration formulae of self-dual (resp. LCD) double $\lambda$-circulant codes. In Section 4, we derive a modified Varshamov-Gilbert bound on the relative distance of the codes considered, building on exact enumeration results. Finally, Section 5 contains conclusions and open problems.

\section{Preliminaries}

\subsection{The ring $R_{k}=\mathbb{F}_{q}[u] /\left\langle u^{k}\right\rangle$}

Let $q$ be a prime power, and let $\mathbb{F}_{q}$ denote the finite field of order $q$. Consider the local ring $R_{k}=\mathbb{F}_{q}[u] /\left\langle u^{k}\right\rangle$ where $u^{k}=0$ with unique maximal ideal $\langle u\rangle$. In double $\lambda$-circulant codes case, we will consider the chain ring $R_{k}=\mathbb{F}_{q}[u] /\left\langle u^{k}\right\rangle$ when it contains a square root of -1 .

Theorem 2.1 If $a_{0}+u a_{1}+\cdots+u^{k-1} a_{k-1} \in R_{k}=\mathbb{F}_{q}[u] /\left\langle u^{k}\right\rangle$ is a square root of -1 if and only if

(1) $q$ is a power of $2, a_{0}^{2}=-1, a_{1}=a_{2}=\cdots=a_{\frac{k-2}{2}}=0$, where $a_{\frac{k}{2}}, a_{\frac{k+2}{2}}, \cdots, a_{k-1} \in$ $\mathbb{F}_{q}$ when $k$ is even; $a_{0}^{2}=-1, a_{1}=a_{2}=\cdots=a_{\frac{k-1}{2}}=0, a_{\frac{k+1}{2}}, a_{\frac{k+2}{2}}, \cdots, a_{k-1} \in \mathbb{F}_{q}$, when $k$ is odd; or

(2) $q=p^{\kappa}$ where $p \equiv 1(\bmod 4)$ or $q=p^{2 \kappa}$ where $p \equiv 3(\bmod 4), a_{0}^{2}=-1, a_{1}=a_{2}=$ $\cdots=a_{k-1}=0$. 
Proof Note that the condition is obviously sufficient. To prove its necessity, when $q$ is a power of 2 , we have $\left(a_{0}+a_{1} u+\cdots+a_{k-1} u^{k-1}\right)^{2}=a_{0}^{2}+a_{1}^{2} u^{2}+\cdots+a_{k-1}^{2} u^{2(k-1)}=$ -1 , when $k$ is even, $a_{0}^{2}=-1, a_{1}=a_{2}=\cdots=a_{\frac{k-2}{2}}=0, a_{\frac{k}{2}}, a_{\frac{k+2}{2}}, \cdots, a_{k-1} \in \mathbb{F}_{q}$; when $k$ is odd, $a_{0}^{2}=-1, a_{1}=a_{2}=\cdots=a_{\frac{k-1}{2}}=0, a_{\frac{k+1}{2}}, a_{\frac{k+2}{2}}, \cdots, a_{k-1} \in \mathbb{F}_{q}$. When $q$ is a power of an odd prime, we have $\left(a_{0}+a_{1} u+\cdots+a_{k-1} u^{k-1}\right)^{2}=a_{0}^{2}+2 a_{0} a_{1} u+\left(2 a_{0} a_{2}+\right.$ $\left.a_{1}^{2}\right) u^{2}+\cdots+\left(a_{0} a_{k-1}+a_{1} a_{k-2}+\cdots+a_{k-1} a_{0}\right) u^{k-1}=-1$, where $a_{i} \in \mathbb{F}_{q}, 0 \leq i \leq k-1$. Then we get $a_{0}^{2}=-1, a_{1}=a_{2}=\cdots=a_{k-1}=0$. Thus $a_{0}$ is a square root of -1 over $\mathbb{F}_{q}$ if and only if $q \equiv 1(\bmod 4)$.

\subsection{Codes}

A linear code $C$ over $R_{k}$ of length $n$ is an $R_{k}$-submodule of $R_{k}^{n}$. If $x=\left(x_{1}, x_{2}, \cdots, x_{n}\right)$ and $y=\left(y_{1}, y_{2}, \cdots, y_{n}\right)$ are two elements of $R_{k}^{n}$, their standard (Euclidean) inner product is defined by

$$
\langle x, y\rangle=\sum_{i=1}^{n} x_{i} y_{i}
$$

and their Hermitian scalar inner product is defined by

$$
\langle x, y\rangle_{H}=\sum_{i=1}^{n} x_{i} \bar{y}_{i}
$$

where the operation is performed in $R_{k}$. For all $z=z_{0}+u z_{1}+\cdots+u^{k-1} z_{k-1} \in \mathbb{F}_{q^{2 Q}}+$ $u \mathbb{F}_{q^{2 Q}}+\cdots+u^{k-1} \mathbb{F}_{q^{2 Q}}$, the conjugation of $z$ over $\mathbb{F}_{q^{2 Q}}+u \mathbb{F}_{q^{2 Q}}+\cdots+u^{k-1} \mathbb{F}_{q^{2 Q}}$ is $\bar{z}=z_{0}^{q^{Q}}+u z_{1}^{q^{Q}}+\cdots+u^{k-1} z_{k-1}^{q^{Q}}$, where $Q$ is a positive integer. The Euclidean (resp. Hermitian) dual code of $C$ is denoted by $C^{\perp}$ (resp. $C^{\perp_{H}}$ ) and defined as $C^{\perp}=\left\{y \in R_{k}^{n} \mid\right.$ $\langle x, y\rangle=0, \forall x \in C\}$ (resp. $C^{\perp_{H}}=\left\{y \in R_{k}^{n} \mid\langle x, y\rangle_{H}=0, \forall x \in C\right\}$ ).

A linear code $C$ of length $n$ over $R_{k}$ is called a self-dual code (resp. Hermitian selfdual code) if $C=C^{\perp}$ (resp. $C=C^{\perp_{H}}$ ). A linear code $C$ of length $n$ over $R_{k}$ is called a linear code with complementary dual (LCD) if $C \bigcap C^{\perp}=\{0\}$ or $C \bigcap C^{\perp_{H}}=\{0\}$.

A matrix $A$ over $R_{k}$ is said to be $\lambda$-circulant if its rows are obtained by successive $\lambda$ shifts from the first row. In this paper, we consider double $\lambda$-circulant codes over $R_{k}$, that is $[2 m, m]$ codes with generator matrices $G=(I, A)$ with $A$ an $m \times m \lambda$-circulant matrix, we can view such a code as an $R_{k}$-module in $R_{k}^{2}$, generated by $(1, h)$ with the first row of $A$ being the $x$-expansion of $h$ in the ring $\frac{R_{k}[x]}{\left\langle x^{m}-\lambda\right\rangle}$.

If $C(m)$ is a family of codes with parameters $\left[m, k_{m}, d_{m}\right]$ over $\mathbb{F}_{q}$, the rate $\rho$ and relative distance $\delta$ are defined as $\rho=\limsup _{m \rightarrow \infty} \frac{k_{m}}{m}$ and $\delta=\liminf _{m \rightarrow \infty} \frac{d_{m}}{m}$, respectively. A family of codes is $\operatorname{good}$ if $\rho \delta>0$.

In number theory, Artin's conjecture on primitive roots states that a given integer $q$ which is neither a perfect square nor -1 is a primitive root modulo infinitely many primes [16]. This was proved conditionally under the Generalized Riemann Hypothesis (GRH) by Hooley [9]. Hence, we can get infinite families of double $\lambda$-circulant codes $C(m)$ over $R_{k}$ where the analysis is made for $x^{m}-1$ with a special factorization.

Recall the $q$-ary entropy function defined for $0 \leq \tilde{t} \leq \frac{q-1}{q}$ by

$$
H_{q}(\tilde{t})= \begin{cases}0, & \text { if } \tilde{t}=0, \\ \tilde{t} \log _{q}(q-1)-\tilde{t} \log _{q}(\tilde{t})-(1-\tilde{t}) \log _{q}(1-\tilde{t}), & \text { if } 0<\tilde{t} \leq \frac{q-1}{q} .\end{cases}
$$


This quantity is instrumental in the estimation of the volume of high-dimensional Hamming balls when the base field is $\mathbb{F}_{q}$. The result we are using is that the volume of the Hamming ball of radius $\tilde{t} m$ is asymptotically equivalent, up to subexponential terms, to $q^{m H_{q}(\tilde{t})}$, when $0<\tilde{t}<1$, and $m$ goes to infinity [10, Lemma 2.10.3].

\subsection{Gray map}

Any integer $z$ can be written uniquely in base $p$ as $z=p_{0}(z)+p p_{1}(z)+p^{2} p_{2}(z)+\cdots$, where $0 \leq p_{i}(z) \leq p-1, i=0,1,2, \ldots$ The Gray map $\Phi: R \rightarrow \mathbb{F}_{p}^{p^{k-1}}$ is defined as follows:

$$
\Phi(a)=\left(b_{0}, b_{1}, b_{2}, \ldots, b_{p^{k-1}-1}\right)
$$

where $a=a_{0}+a_{1} u+\cdots+a_{k-1} u^{k-1}$. Then for all $0 \leq i \leq p^{k-2}-1,0 \leq \tau \leq p-1$, we have

$$
b_{i p+\tau}=\left\{\begin{array}{lr}
a_{k-1}+\sum_{l=1}^{k-2} p_{l-1}(i) a_{l}+\tau a_{0}, & \text { if } k \geq 3, \\
a_{1}+\tau a_{0}, & \text { if } k=2 .
\end{array}\right.
$$

Note that, more generally, Gray maps have been defined at the level of finite chain rings in $[15,23]$, linking codes over rings to codes over finite fields. For instance, when $p=$ $k=2$, it is easy to check that the Gray map adopted in the trace codes of [21] is the same as the Gray map defined here. As an additional example, when $p=k=3$, write $\Phi\left(a_{0}+a_{1} u+a_{2} u^{2}\right)=\left(b_{0}, b_{1}, b_{2}, \cdots, b_{8}\right)$. According to the definition above, we have $0 \leq i \leq 2,0 \leq \tau \leq 2$ and $\sum_{l=1}^{k-2} p_{l-1}(i) a_{l}=p_{0}(i) a_{1}=i a_{1}$. Then we get

$$
\begin{gathered}
b_{0}=a_{2}, b_{1}=a_{2}+a_{0}, b_{2}=a_{2}+2 a_{0}, b_{3}=a_{2}+a_{1}, b_{4}=a_{2}+a_{1}+a_{0}, \\
b_{5}=a_{2}+a_{1}+2 a_{0}, b_{6}=a_{2}+2 a_{1}, b_{7}=a_{2}+2 a_{1}+a_{0}, b_{8}=a_{2}+2 a_{1}+2 a_{0} .
\end{gathered}
$$

It is easy to extend the Gray map from $R_{k}^{m}$ to $\mathbb{F}_{p}^{p^{k-1} m}$, and we also know from [22] that $\Phi$ is injective and linear.

\section{Algebraic structure of $\lambda$-circulant codes of index two}

In this section, we study the exact enumeration of the double self-dual and LCD $\lambda$-circulant codes over $R_{k}$.

\subsection{Double circulant codes $(\lambda=1)$}

In this subsection, we assume $m$ is an odd integer and $\operatorname{gcd}(m, q)=1$. We can cast the factorization of $x^{m}-1$ into distinct basic irreducible polynomials over $R_{k}=\mathbb{F}_{q}[u] /\left\langle u^{k}\right\rangle$ in the form

$$
x^{m}-1=\delta(x-1) \prod_{i=2}^{s} g_{i}(x) \prod_{j=1}^{t} h_{j}(x) h_{j}^{*}(x)
$$


where $\delta$ is a unit in $R_{k}$, the polynomial $g_{i}(x)$ is self-reciprocal of degree $2 e_{i}$ for $2 \leq i \leq s$, and $h_{j}^{*}(x)$ is the reciprocal polynomial of $h_{j}(x)$ with degree $d_{j}$ for $1 \leq j \leq t$. By the Chinese Remainder Theorem (CRT), we have

$$
\begin{aligned}
\frac{R_{k}[x]}{\left\langle x^{m}-1\right\rangle} \simeq & \frac{R_{k}[x]}{\langle x-1\rangle} \oplus\left(\bigoplus_{i=2}^{s} R_{k}[x] /\left\langle g_{i}(x)\right\rangle\right) \oplus\left(\bigoplus_{j=1}^{t}\left(R_{k}[x] /\left\langle h_{j}(x)\right\rangle \oplus R_{k}[x] /\left\langle h_{j}^{*}(x)\right\rangle\right)\right) \\
\simeq & \frac{\mathbb{F}_{q}[u, x]}{\left\langle u^{k}, x-1\right\rangle} \oplus\left(\bigoplus_{i=2}^{s} \frac{\mathbb{F}_{q}[u, x]}{\left\langle u^{k}, g_{i}(x)\right\rangle}\right) \oplus\left(\bigoplus_{j=1}^{t}\left(\frac{\mathbb{F}_{q}[u, x]}{\left\langle u^{k}, h_{j}(x)\right\rangle} \oplus \frac{\mathbb{F}_{q}[u, x]}{\left\langle u^{k}, h_{j}^{*}(x)\right\rangle}\right)\right) \\
\simeq & R_{k} \oplus\left(\bigoplus_{i=2}^{s}\left(\mathbb{F}_{q^{2 e_{i}}}+u \mathbb{F}_{q^{2 e_{i}}}+\cdots+u^{k-1} \mathbb{F}_{q^{2 e_{i}}}\right)\right) \oplus\left(\bigoplus _ { j = 1 } ^ { t } \left(\left(\mathbb{F}_{q^{d_{j}}}+u \mathbb{F}_{q^{d_{j}}}+\cdots\right.\right.\right. \\
& \left.\left.\left.+u^{k-1} \mathbb{F}_{q^{d_{j}}}\right) \oplus\left(\mathbb{F}_{q^{d_{j}}}+u \mathbb{F}_{q^{d_{j}}}+\cdots+u^{k-1} \mathbb{F}_{q^{d_{j}}}\right)\right)\right) \\
:= & R_{k} \oplus\left(\bigoplus_{i=2}^{s} R_{k\left(2 e_{i}\right)}\right) \oplus\left(\bigoplus_{j=1}^{t}\left(R_{k\left(d_{j}\right)} \oplus R_{k\left(d_{j}\right)}\right)\right) .
\end{aligned}
$$

Note that all of these rings are extensions of $R_{k}$. This decomposition naturally extends to $\left(\frac{R_{k}[x]}{\left\langle x^{m}-1\right\rangle}\right)^{2}$ as

$$
\left(\frac{R_{k}[x]}{\left\langle x^{m}-1\right\rangle}\right)^{2} \simeq R_{k} \oplus\left(\bigoplus_{i=2}^{s} R_{k\left(2 e_{i}\right)}^{2}\right) \oplus\left(\bigoplus_{j=1}^{t}\left(R_{k\left(d_{j}\right)}^{2} \oplus R_{k\left(d_{j}\right)}^{2}\right)\right) .
$$

In particular, each linear code $C$ of length 2 over $\frac{R_{k}[x]}{\left\langle x^{m}-1\right\rangle}$ can be decomposed as the "CRT sum"

$$
C \simeq C_{1} \oplus\left(\bigoplus_{i=2}^{s} C_{i}\right) \oplus\left(\bigoplus_{j=1}^{t}\left(C_{j}^{\prime} \oplus C_{j}^{\prime \prime}\right)\right),
$$

where $C_{1}$ is a linear code over $R_{k}$ of length $2, C_{i}$ is a linear code over $R_{k\left(2 e_{i}\right)}$ of length 2 for each $2 \leq i \leq s$, and $C_{j}^{\prime}$ and $C_{j}^{\prime \prime}$ are linear codes over $R_{k\left(d_{j}\right)}$ of length 2 for each $1 \leq j \leq t$, which are called the constituents of $C$.

Lemma 3.1 Keep the same notations as above, then

(1) $C_{1}$ is LCD if and only if $1+r^{2} \in R_{k}^{\times}$with $C_{1}=\langle(1, r)\rangle$;

(2) $C_{i}$ is LCD if and only if $1+\eta \bar{\eta} \in R_{k\left(2 e_{i}\right)}^{\times}$with $C_{i}=\langle(1, \eta)\rangle$;

(3) $C_{j}^{\prime} \oplus C_{j}^{\prime \prime}$ are LCD if and only if $1+\eta^{\prime} \eta^{\prime \prime} \in R_{k\left(d_{j}\right)}^{\times}$with $C_{j}^{\prime}=\left\langle\left(1, \eta^{\prime}\right)\right\rangle$ and $C_{j}^{\prime \prime}=$ $\left\langle\left(1, \eta^{\prime \prime}\right)\right\rangle$.

Proof (1) " $\Longrightarrow$ " If $C_{1}$ is LCD, suppose $1+r^{2} \notin R_{k}^{\times}$, then $1+r^{2} \in\langle u\rangle$. We have $u^{k-1}\left(1+r^{2}\right)=0$,i.e., $\left\langle u^{k-1}(1, r),(1, r)\right\rangle=0$, then $u^{k-1}(1, r) \in C_{1}^{\perp}$, which implies $u^{k-1}(1, r) \in C_{1} \cap C_{1}^{\perp}$, a contradiction. 


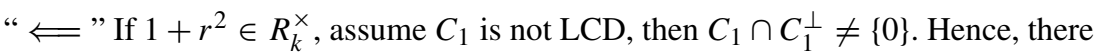
exists $r^{\prime} \in R_{k}^{\times}$such that $r^{\prime}(1, r) \in C_{1} \cap C_{1}^{\perp}$, then $\left\langle r^{\prime}(1, r),(1, r)\right\rangle=r^{\prime}\left(1+r^{2}\right)=$ 0 , since $1+r^{2} \in R_{k}^{\times}$, then $r^{\prime}=0$, a contradiction.

(2) “ $\Longrightarrow$ " If $C_{i}$ is LCD, suppose $1+\eta \bar{\eta} \notin R_{k\left(2 e_{i}\right)}^{\times}$, then $1+\eta \bar{\eta} \in\langle u\rangle$. We have $u^{k-1}(1+\eta \bar{\eta})=0$,i.e., $\left\langle u^{k-1}(1, \eta),(1, \eta)\right\rangle_{H}=0$, then $u^{k-1}(1, \eta) \in C_{i}^{\perp_{H}}$, which implies $u^{k-1}(1, \eta) \in C_{i} \cap C_{i}^{\perp_{H}}$, a contradiction.

" $\Longleftarrow$ If $1+\eta \bar{\eta} \in R_{k\left(2 e_{i}\right)}^{\times}$, assume $C_{i}$ is not LCD, then $C_{i} \cap C_{i}^{\perp_{H}} \neq\{0\}$. If $\eta \in R_{k\left(2 e_{i}\right)}^{\times}$, then $C_{i}^{\perp_{H}}=\left\langle\left(1,-\frac{1}{\bar{\eta}}\right)\right\rangle$, there exist $k_{1}, k_{2} \in R_{k\left(2 e_{i}\right)}^{\times}$such that $k_{1}(1, \eta)=$ $k_{2}\left(1,-\frac{1}{\bar{\eta}}\right)$, i.e., $k_{1}(1+\eta \bar{\eta})=0$. And $1+\eta \bar{\eta} \in R_{k\left(2 e_{i}\right)}^{\times}$, then $k_{1}=0$, a contradiction. If $\eta \in\langle u\rangle$, we can let $\eta=u a_{1}+u^{2} a_{2}+\cdots+u^{k-1} a_{k-1}, a_{i} \in \mathbb{F}_{q^{2 e_{i}}}, 0 \leq i \leq k-1$, then the generator matrix of $C_{i}^{\perp_{H}}$ is of the form

$$
\left(\begin{array}{cc}
-\left(u \bar{a}_{1}+u^{2} \bar{a}_{2}+\cdots+u^{k-1} \bar{a}_{k-1}\right) & 1 \\
0 & u^{k-1} k_{3}
\end{array}\right),
$$

where $k_{3} \in \mathbb{F}_{q^{2 e_{i}}}$. Thus, there exist $k_{4}, k_{5}, k_{6} \in R_{k\left(2 e_{i}\right)}^{\times}$such that $k_{4}\left(1, u a_{1}+u^{2} a_{2}+\right.$ $\left.\cdots+u^{k-1} a_{k-1}\right)=k_{5}\left(-\left(u \bar{a}_{1}+u^{2} \bar{a}_{2}+\cdots+u^{k-1} \bar{a}_{k-1}\right), 1\right)+k_{6}\left(0, u^{k-1} k_{3}\right)$, we then obtain

$$
\left\{\begin{array}{l}
k_{4}=-\left(u \bar{a}_{1}+u^{2} \bar{a}_{2}+\cdots+u^{k-1} \bar{a}_{k-1}\right) k_{5}, \\
\left(u a_{1}+u^{2} a_{2}+\cdots+u^{k-1} a_{k-1}\right) k_{4}=k_{5}+u^{k-1} k_{3} k_{6},
\end{array}\right.
$$

by (2), we get $k_{4} \in R_{k\left(2 e_{i}\right)}^{\times}$, but $-\left(u \bar{a}_{1}+u^{2} \bar{a}_{2}+\cdots+u^{k-1} \bar{a}_{k-1}\right) k_{5} \in\langle u\rangle$, a contradiction.

(3) “ $\Longrightarrow$ " If $C_{j}^{\prime} \oplus C_{j}^{\prime \prime}$ is LCD, assume $1+\eta^{\prime} \eta^{\prime \prime} \notin R_{k\left(d_{j}\right)}^{\times}$, then $1+\eta^{\prime} \eta^{\prime \prime} \in\langle u\rangle$. We have $u^{k-1}\left(1+\eta^{\prime} \eta^{\prime \prime}\right)=0$,i.e., $\left\langle u^{k-1}\left(1, \eta^{\prime}\right),\left(1, \eta^{\prime \prime}\right)\right\rangle=0$, then $u^{k-1}\left(1, \eta^{\prime}\right) \in C_{j}^{\prime \prime}$ (or $\left.u^{k-1}\left(1, \eta^{\prime \prime}\right) \in C_{j}^{\prime \perp}\right)$, which implies $u^{k-1}\left(1, \eta^{\prime}\right) \in C_{j}^{\prime} \cap C_{j}^{\prime \prime}\left(\right.$ or $u^{k-1}\left(1, \eta^{\prime \prime}\right) \in$ $\left.C_{j}^{\prime \prime} \cap C_{j}^{\prime \perp}\right)$, a contradiction.

“ $\Longleftarrow$ If $1+\eta^{\prime} \eta^{\prime \prime} \in R_{k\left(d_{j}\right)}^{\times}$, assume $C_{j}^{\prime} \oplus C_{j}^{\prime \prime}$ is not LCD, then

$$
\left\{\begin{array}{l}
C_{j}^{\prime} \cap C_{j}^{\prime \prime \perp} \neq\{0\}, \\
C_{j}^{\prime \prime} \cap C_{j}^{\prime \perp} \neq\{0\} .
\end{array}\right.
$$

If $C_{j}^{\prime} \cap C_{j}^{\prime \prime \perp} \neq\{0\}$, then there exists $k^{\prime} \in R_{k\left(d_{j}\right)}^{\times}$such that $k^{\prime}\left(1, \eta^{\prime}\right) \in C_{j}^{\prime} \cap C_{j}^{\prime \prime \perp}$,i.e., $\left\langle k^{\prime}\left(1, \eta^{\prime}\right),\left(1, \eta^{\prime \prime}\right)\right\rangle=k^{\prime}\left(1+\eta^{\prime} \eta^{\prime \prime}\right)=0$, a contradiction.

Theorem 3.2 Let $m$ denote a positive odd integer, and $q$ a prime coprime with $m$. If $x^{m}-1$ can be factored into irreducible polynomials over $R_{k}$ as in (1), where $m=1+\sum_{i=2}^{s} 2 e_{i}+$ $2 \sum_{j=1}^{t} d_{j}$. Then

(1) the total number of self-dual double circulant codes over $R_{k}$ is

$$
B \prod_{i=2}^{s}\left(q^{e_{i}}+1\right) q^{e_{i}(k-1)} \prod_{j=1}^{t}\left(q^{d_{j}}-1\right) q^{d_{j}(k-1)} \text {, where }
$$

1) when $q$ is a power of $2, B=2 q^{\frac{k}{2}}, k$ is even, or $B=2 q^{\frac{k-1}{2}}, k$ is odd; 
2) when $q$ is a power of odd prime, $B=2$.

(2) the total number of LCD double circulant codes over $R_{k}$ is

$$
(q-2) q^{k-1} \prod_{i=2}^{s}\left(q^{2 e_{i}}-\left(q^{e_{i}}+1\right)\right) q^{2(k-1) e_{i}} \prod_{j=1}^{t}\left(q^{2 k d_{j}}-q^{2(k-1) d_{j}}\left(q^{d_{j}}-1\right)\right) .
$$

Proof (1) We can count the number of self-dual double circulant codes by counting their constituent codes.

Let $(1, r)$ be the generator of the self-dual code $C_{1}$ over $R_{k}$. By Theorem 2.1, when $q$ is a power of 2 , the number of $r$ is equal to $2 q^{\frac{k}{2}}$, where $k$ is even $\left(2 q^{\frac{k-1}{2}}\right.$, where $k$ is odd); when $q$ is a power of odd prime, the number of choices for $r$ is equal to 2 .

Let $\left(1, c_{e_{i}}\right)$ be the generators of Hermitian self-dual codes $C_{i}$ over $R_{k\left(2 e_{i}\right)}, 2 \leq i \leq$ $s$, then $\left\langle\left(1, c_{e_{i}}\right),\left(1, c_{e_{i}}\right)\right\rangle_{H}=1+c_{e_{i}} \bar{c}_{e_{i}}=0$. Let $c_{e_{i}}=c_{0}+u c_{1}+\cdots+u^{k-1} c_{k-1}$, where $c_{\ell} \in \mathbb{F}_{q^{2 e_{i}}}, 0 \leq \ell \leq k-1$, we then have

$$
\left\{\begin{array}{l}
c_{0} c_{0}^{q^{e_{i}}}=-1 \\
c_{0} c_{1}^{q^{e_{i}}}+c_{1} c_{0}^{q^{e_{i}}}=0 \\
c_{0} c_{2}^{q^{e_{i}}}+c_{1} c_{1}^{q^{e_{i}}}+c_{2} c_{0}^{q^{e_{i}}}=0 \\
c_{0} c_{3}^{q^{e_{i}}}+c_{1} c_{2}^{q^{e_{i}}}+c_{2} c_{1}^{q^{e_{i}}}+c_{3} c_{0}^{q^{e_{i}}}=0 \\
c_{0} c_{4}^{q^{e_{i}}}+c_{1} c_{3}^{q^{e_{i}}}+c_{2} c_{2}^{q^{e_{i}}}+c_{3} c_{1}^{q^{e_{i}}}+c_{4} c_{0}^{q^{e_{i}}}=0 \\
\vdots \\
c_{0} c_{k-1}^{q^{e_{i}}}+c_{1} c_{k-2}^{q^{e_{i}}}+\cdots+c_{k-1} c_{0}^{q^{e_{i}}}=0
\end{array}\right.
$$

$$
\Longleftrightarrow\left\{\begin{array}{l}
\operatorname{Norm}\left(c_{0}\right)=-1, \\
\operatorname{Tr}\left(c_{0} c_{1}^{q^{e_{i}}}\right)=0, \\
\operatorname{Tr}\left(c_{0} c_{2}^{q^{e_{i}}}\right)+\operatorname{Norm}\left(c_{1}\right)=0, \\
\operatorname{Tr}\left(c_{0} c_{3}^{q^{e_{i}}}\right)+\operatorname{Tr}\left(c_{1} c_{2}^{q^{e_{i}}}\right)=0, \\
\operatorname{Tr}\left(c_{0} c_{4}^{q^{e_{i}}}\right)+\operatorname{Tr}\left(c_{1} c_{3}^{q^{e_{i}}}\right)+\operatorname{Norm}\left(c_{2}\right)=0, \\
\vdots \\
\operatorname{Tr}\left(c_{0} c_{k-1}^{q^{e_{i}}}\right)+\operatorname{Tr}\left(c_{1} c_{k-2}^{q^{e_{i}}}\right)+\cdots+\operatorname{Tr}\left(c_{\frac{k-2}{2}} c_{\frac{k}{2}}\right)=0, \text { when } k \text { is even, or } \\
\operatorname{Tr}\left(c_{0} c_{k-1}^{q^{e_{i}}}\right)+\operatorname{Tr}\left(c_{1} c_{k-2}^{q_{e_{i}}}\right)+\cdots+\operatorname{Tr}\left(c_{\frac{k-3}{2}} c_{\frac{k+1}{2}}\right)+\operatorname{Norm}\left(c_{\frac{k-1}{2}}\right)=0, \text { when } k \text { is odd, }
\end{array}\right.
$$

where the $\operatorname{Norm}()$ and $\operatorname{Tr}()$ are maps norm and trace from $\mathbb{F}_{q^{2 e_{i}}}$ to $\mathbb{F}_{q^{e_{i}}}$. So there are $q^{e_{i}}+1$ roots for $\operatorname{Norm}\left(c_{0}\right)=-1$ and $q^{e_{i}}$ choices for $c_{i}$ for $1 \leq \ell \leq k-1$. Clearly, the number of solutions of (3) is equal to $\left(q^{e_{i}}+1\right) q^{e_{i}(k-1)}$.

As for reciprocal pairs, note that a pair $\left(h_{j}(x), h_{j}^{*}(x)\right)$ both of degree $d_{j}$ leads to counting dual pairs of codes (for the Euclidean inner product) of length 2 over $R_{k\left(d_{j}\right)}$, that is to count the number of solutions of $1+c_{d_{j}}^{\prime} c_{d_{j}}^{\prime \prime}=0$, where $\left(1, c_{d_{j}}^{\prime}\right)$ and $\left(1, c_{d_{j}}^{\prime \prime}\right)$ are the generators of $C_{j}^{\prime}$ and $C_{j}^{\prime \prime}$, respectively. If $c_{d_{j}}^{\prime} \in R_{k\left(d_{j}\right)}^{\times}$, then $c_{d_{j}}^{\prime \prime}=-\frac{1}{c_{d_{j}}^{\prime}}$, there are $\left|R_{k\left(d_{j}\right)}^{\times}\right|=\left(q^{d_{j}}-1\right) q^{d_{j}(k-1)}$ choices for $\left(c_{d_{j}}^{\prime}, c_{d_{j}}^{\prime \prime}\right)$. If $c_{d_{j}}^{\prime} \in R_{k\left(d_{j}\right)} \backslash R_{k\left(d_{j}\right)}^{\times}$, then $c_{d_{j}}^{\prime}=u x_{1}+u^{2} x_{2}+\cdots+u^{k-1} x_{k-1} \in\langle u\rangle$. In this case, $1+c_{d_{j}}^{\prime} c_{d_{j}}^{\prime \prime}=0$, which is impossible.

(2) The code $C_{1}$ is an LCD code, by Lemma 3.1 (1), we can get $1+r^{2} \in R_{k}^{\times}$. Let $r=$ $r_{0}+u r_{1}+\cdots+u^{k-1} r_{k-1} \in R_{k}$, then $1+r^{2}=1+\left(r_{0}+u r_{1}+u^{2} r_{2}+\cdots+u^{k-1} r_{k-1}\right)^{2}=$ 
$1+r_{0}^{2}+2 r_{0} r_{1} u+\left(2 r_{0} r_{2}+r_{1}^{2}\right) u^{2}+\cdots+\left(r_{0} r_{k-1}+r_{1} r_{k-2}+\cdots+r_{k-1} r_{0}\right) u^{k-1} \in R_{k}^{\times}$. Hence, the number of $r$ is equal to $(q-2) q^{k-1}$.

The code $C_{i}$ is an LCD code, by Lemma 3.1 (2), we can get $1+\eta \bar{\eta} \in R_{k\left(2 e_{i}\right)}^{\times}$. Let $\eta=\eta_{0}+u \eta_{1}+\cdots+u^{k-1} \eta_{k-1}$, then $1+\eta \bar{\eta}=1+\left(\eta_{0}+u \eta_{1}+\cdots+u^{k-1} \eta_{k-1}\right)\left(\eta_{0}^{q^{e_{i}}}+\right.$ $\left.u \eta_{1}^{q^{e_{i}}}+\cdots+u^{k-1} \eta_{k-1}^{q^{e_{i}}}\right)=1+\eta_{0}^{q^{e_{i}}+1}+u\left(\eta_{0} \eta_{1}^{q^{e_{i}}}+\eta_{1} \eta_{0}^{q^{e_{i}}}\right)+\cdots+u^{k-1}\left(\eta_{0} \eta_{k-1}^{q^{e_{i}}}+\right.$ $\left.\eta_{1} \eta_{k-2}^{q_{i}{ }^{e_{i}}}+\cdots+\eta_{k-1} \eta_{0}^{q^{e_{i}}}\right) \in R_{k\left(2 e_{i}\right)}^{\times}$. Hence, the number of $\eta$ is equal to $\left(q^{2 e_{i}}-\left(q^{e_{i}}+\right.\right.$ 1)) $q^{2(k-1) e_{i}}$.

Next, we count the number of LCD double circulant codes of length 2 over $R_{k\left(d_{j}\right)}$ for the pairs $h_{j}(x)$ and $h_{j}^{*}(x)$ with $\operatorname{deg}\left(h_{j}(x)\right)=\operatorname{deg}\left(h_{j}^{*}(x)\right)=d_{j}$. By Lemma 3.1 (3), we then get

$$
\left\{\begin{array}{l}
C_{j}^{\prime} \cap C_{j}^{\prime \prime \perp}=\{0\}, \\
C_{j}^{\prime \prime} \cap C_{j}^{\prime \perp}=\{0\} .
\end{array} \Longleftrightarrow 1+\eta^{\prime} \eta^{\prime \prime} \in R_{k\left(d_{j}\right)}^{\times} .\right.
$$

Without loss of generality, we discuss on the unit character of $\eta^{\prime}$ as follows.

1) If $\eta^{\prime} \in R_{k\left(d_{j}\right)}^{\times}$, then $\eta^{\prime \prime} \in-\frac{1}{\eta^{\prime}}+R_{k\left(d_{j}\right)}^{\times}$and $\left|\frac{-1}{\eta^{\prime}}+R_{k\left(d_{j}\right)}^{\times}\right|=\left|R_{k\left(d_{j}\right)}^{\times}\right|=q^{(k-1) d_{j}}\left(q^{d_{j}}-1\right)$. Hence, there are $\left|R_{k\left(d_{j}\right)}^{\times}\right|^{2}=q^{2(k-1) d_{j}}\left(q^{d_{j}}-1\right)^{2}$ choices for $\left(\eta^{\prime}, \eta^{\prime \prime}\right)$.

2) If $\eta^{\prime} \in R_{k\left(d_{j}\right)} \backslash\left\{R_{k\left(d_{j}\right)}^{\times} \cup\{0\}\right\}$, let $\eta^{\prime \prime}=\eta_{0}^{\prime \prime}+u \eta_{1}^{\prime \prime}+\cdots+u^{k-1} \eta_{k-1}^{\prime \prime}$, then $\eta^{\prime}=u \eta_{1}^{\prime}+u^{2} \eta_{2}^{\prime}+$ $\cdots+u^{k-1} \eta_{k-1}^{\prime}$, where $\eta_{\ell_{1}}^{\prime}$ can't be all zero, $1 \leq \ell_{1} \leq k-1, \eta_{\ell_{2}}^{\prime \prime} \in \mathbb{F}_{q^{d_{j}}}, 0 \leq \ell_{2} \leq k-1$. We then have $1+\eta^{\prime} \eta^{\prime \prime}=1+u \eta_{1}^{\prime} \eta_{0}^{\prime \prime}+u^{2}\left(\eta_{1}^{\prime} \eta_{1}^{\prime \prime}+\eta_{2}^{\prime} \eta_{0}^{\prime \prime}\right)+\cdots+u^{k-1}\left(\eta_{1}^{\prime} \eta_{k-2}^{\prime \prime}+\eta_{2}^{\prime} \eta_{k-3}^{\prime \prime}+\right.$ $\left.\cdots+\eta_{k-1}^{\prime} \eta_{0}^{\prime \prime}\right) \in R_{k\left(d_{j}\right)}^{\times}$. Thus, there are $\left(q^{(k-1) d_{j}}-1\right) q^{k d_{j}}$ choices for $\left(\eta^{\prime}, \eta^{\prime \prime}\right)$.

3) If $\eta^{\prime}=0$, then $\eta^{\prime \prime} \in R_{k\left(d_{j}\right)}$, thus there are $q^{k d_{j}}$ choices for $\eta^{\prime \prime}$.

Hence, the number of the last case about reciprocal pairs is $q^{2(k-1) d_{j}}\left(q^{d_{j}}-1\right)^{2}+\left(q^{(k-1) d_{j}}-\right.$ 1) $q^{k d_{j}}+q^{k d_{j}}=q^{2 k d_{j}}-q^{2(k-1) d_{j}}\left(q^{d_{j}}-1\right)$. The proof of the theorem is now completed.

\subsection{Double negacirculant codes $(\lambda=-1)$}

In this subsection, assume $m$ is an even integer and $\operatorname{gcd}(m, q)=1$, where $q$ is a prime power. We can cast the factorization of $x^{m}+1$ into distinct basic irreducible polynomials over $R_{k}$ as follows.

$$
x^{m}+1=\epsilon \prod_{i=1}^{s} g_{i}(x) \prod_{j=1}^{t} h_{j}(x) h_{j}^{*}(x),
$$

where $\epsilon \in R_{k}^{\times}, g_{i}(x)=g_{i}^{*}(x)$ with $\operatorname{deg}\left(g_{i}(x)\right)=2 e_{i}, 1 \leq i \leq s$, and $h_{j}^{*}(x)$ is the reciprocal polynomial of $h_{j}(x)$ with $\operatorname{deg}\left(h_{j}(x)\right)=\operatorname{deg}\left(h_{j}^{*}(x)\right)=d_{j}, 1 \leq j \leq t$. Using the same notations and argument as in Subsection 3.1, we can easily carry out the result as follows:

$$
\frac{R_{k}[x]}{\left\langle x^{m}+1\right\rangle} \simeq\left(\bigoplus_{i=1}^{s} R_{k\left(2 e_{i}\right)}\right) \oplus\left(\bigoplus_{j=1}^{t}\left(R_{k\left(d_{j}\right)} \oplus R_{k\left(d_{j}\right)}\right)\right),
$$

and

$$
C \simeq\left(\bigoplus_{i=1}^{s} C_{i}\right) \oplus\left(\bigoplus_{j=1}^{t}\left(C_{j}^{\prime} \oplus C_{j}^{\prime \prime}\right)\right)
$$


Theorem 3.3 Let $m$ denote a positive even integer, and $q$ a prime power coprime with $m$. The factorization of $x^{m}+1$ over $R_{k}$ is of the form (4) with $m=\sum_{i=1}^{s} 2 e_{i}+2 \sum_{j=1}^{t} d_{j}$. Then

(1) the total number of self-dual double negacirculant codes over $R_{k}$ is

$$
\prod_{i=1}^{s}\left(q^{e_{i}}+1\right) q^{e_{i}(k-1)} \prod_{j=1}^{t}\left(q^{d_{j}}-1\right) q^{d_{j}(k-1)} .
$$

(2) the total number of LCD double negacirculant codes over $R_{k}$ is

$$
\prod_{i=1}^{s}\left(q^{2 e_{i}}-\left(q^{e_{i}}+1\right)\right) q^{2(k-1) e_{i}} \prod_{j=1}^{t}\left(q^{2 k d_{j}}-q^{2(k-1) d_{j}}\left(q^{d_{j}}-1\right)\right) .
$$

Proof This proof is similar to that of Theorem 3.2, so we omitted it here.

Now, we consider a special factorization of $x^{m}+1$, where $m$ is a power of $2, q$ is an odd prime. According to [17, Theorem 1] and [2, Theorems 5.1,5.3], we know that $x^{m}+1$ can be factored into two (resp. four) basic irreducible polynomials, which are reciprocal of each other over $R_{k}$, by limiting the size of $\triangle$ and $U$, because $\mathbb{F}_{q}$ is a subring of $R_{k}$. We can get the following lemma.

Lemma 3.4 Let $m$ be a power of $2, q \equiv \pm 1(\bmod 4)$.

(1) If $q=2^{2} e \pm 1$, e is odd, then $x^{m}+1$ factors into two basic irreducible polynomials over $R_{k}$ as follows.

$$
x^{m}+1=h(x) h^{*}(x)
$$

with $\operatorname{deg}(h(x))=\operatorname{deg}\left(h^{*}(x)\right)=\frac{m}{2}$. In this case, the number of self-dual (resp. LCD) double negacirculant codes over $R_{k}$ is

$$
\left.\left(q^{\frac{m}{2}}-1\right) q^{\frac{m(k-1)}{2}}\left(\text { resp. } q^{k m}-q^{m(k-1)}\left(q^{\frac{m}{2}}-1\right)\right)\right) .
$$

(2) If $q=2^{3} e \pm 1$, e is odd, then $x^{m}+1$ factors into four basic irreducible polynomials over $R_{k}$ as follows.

$$
x^{m}+1=h_{1}(x) h_{1}^{*}(x) h_{2}(x) h_{2}^{*}(x)
$$

with $\operatorname{deg}\left(h_{1}(x)\right)=\operatorname{deg}\left(h_{1}^{*}(x)\right)=\operatorname{deg}\left(h_{2}(x)\right)=\operatorname{deg}\left(h_{2}^{*}(x)\right)=\frac{m}{4}$. In this case, the number of self-dual (resp. LCD) double negacirculant codes over $R_{k}$ is

$$
\left(q^{\frac{m}{4}}-1\right)^{2} q^{\frac{m(k-1)}{2}}\left(\operatorname{resp} \cdot\left(q^{\frac{k m}{2}}-q^{\frac{m(k-1)}{2}}\left(q^{\frac{m}{4}}-1\right)\right)^{2}\right) .
$$

\subsection{Quasi-twisted codes of index two $\left(\lambda=1+\omega u^{\mathfrak{t}}\right)$}

In this subsection, we focus on the case $\left(1+\omega u^{\mathfrak{t}}\right)=\left(1+\omega u^{\mathfrak{t}}\right)^{-1}=\left(1-\omega u^{\mathfrak{t}}\right)$. According to [19], $x^{m}-\left(1+\omega u^{\mathfrak{t}}\right)$ can be uniquely expressed as

$$
x^{m}-\left(1+\omega u^{\mathfrak{t}}\right)=\varsigma g_{1}(x) \prod_{i=2}^{s} g_{i}(x) \prod_{j=1}^{t} h_{j}(x) h_{j}^{*}(x)
$$

where $m$ is an odd, then $g_{1}(x)=x-\left(1+\omega u^{\mathfrak{t}}\right), \varsigma \in R_{k}^{\times}, g_{i}(x)=g_{i}^{*}(x)$ with $\operatorname{deg}\left(g_{i}(x)\right)=2 e_{i}, 2 \leq i \leq s$, and $h_{j}^{*}(x)$ is the reciprocal polynomial of $h_{j}(x)$ with $\operatorname{deg}\left(h_{j}(x)\right)=\operatorname{deg}\left(h_{j}^{*}(x)\right)=d_{j}, 1 \leq j \leq t$. 
In fact, we notice that a $\left(1+\omega u^{\mathrm{t}}\right)$-QT code over $R_{k}$ is self-dual only if $1+\omega u^{\mathrm{t}}=1-\omega u^{\mathrm{t}}$, i.e., $2 \omega u^{\mathfrak{t}}=0 \Longrightarrow \operatorname{char}\left(R_{k}\right)=2$ over $R_{k}$.

Conjecture 3.5 Assume that $m$ is an odd prime and $\operatorname{gcd}(m, q)=1$, where $q$ is a prime power. Let $\alpha \mid(m-1)$ and $\operatorname{ord}_{m}(q)=\frac{m-1}{\alpha}$, we can cast the factorization of $x^{m}-\lambda$ into distinct basic irreducible polynomials over $R_{k}=\frac{\mathbb{F}_{q}[u]}{\left\langle u^{k}\right\rangle}$ as follows.

(1) If $\alpha$ is an odd integer, then we have $x^{m}-\lambda=A(x) \prod_{i=1}^{\alpha} g_{i}(x)$, where $g_{i}(x)=g_{i}^{*}(x)$, $\operatorname{deg}\left(g_{i}(x)\right)=\frac{m-1}{\alpha}$

(2) If $\alpha$ is an even integer, then we have $x^{m}-\lambda=A(x) \prod_{j=1}^{\frac{\alpha}{2}} h_{j}(x) h_{j}^{*}(x)$, where $\operatorname{deg}\left(h_{j}(x)\right)=\operatorname{deg}\left(h_{j}^{*}(x)\right)=\frac{m-1}{\alpha} ;$ if

(i) $\lambda=1, A(x)=x-1$, or

(ii) $\lambda=-1, A(x)=x+1$, or

(iii) $\lambda=1+\omega u^{\mathfrak{t}}, q$ is a power of $2, A(x)=x+1+\omega u^{\mathfrak{t}}$, where $\mathfrak{t} \geq\left\lceil\frac{k}{2}\right\rceil, \omega \in R_{k}^{\times}$.

Now, we only give some examples to illustrate its correctness. In fact, we have tried a lot of examples by Magma, the conjecture is also correct. But we fail to prove it. Thus we would like to put it here as a conjecture.

Example 3.6 Let $R_{k}=\mathbb{F}_{3}[u] /\left\langle u^{k}\right\rangle, m=11, \alpha=2$ be an even integer, implies $\alpha \mid(m-$ $1)=2 \mid 10, \operatorname{ord}_{11}(3)=\frac{m-1}{\alpha}=5$, then by Conjecture 3.5,

$$
\begin{aligned}
& x^{11}-1=(x-1)\left(x^{5}+2 x^{3}+x^{2}+2 x+2\right)\left(x^{5}+x^{4}+2 x^{3}+x^{2}+2\right), \\
& x^{11}+1=(x+1)\left(x^{5}+2 x^{3}+2 x^{2}+2 x+1\right)\left(x^{5}+2 x^{4}+2 x^{3}+2 x^{2}+1\right) .
\end{aligned}
$$

Example 3.7 Let $R_{k}=\mathbb{F}_{2}[u] /\left\langle u^{k}\right\rangle, m=5, u^{k}=0, \mathfrak{t} \geq\left\lceil\frac{k}{2}\right\rceil, \alpha=1$ be an odd integer, implies $\alpha|(m-1)=1| 4, \operatorname{ord}_{5}(2)=\frac{m-1}{\alpha}=4$, then by Conjecture 3.5,

$$
\begin{aligned}
x^{5}-1 & =(x-1)\left(x^{4}+x^{3}+x^{2}+x+1\right), \\
x^{5}-\left(1+u^{\mathfrak{t}}\right) & =\left(x+1+u^{\mathfrak{t}}\right)\left(x^{4}+\left(1+u^{\mathfrak{t}}\right) x^{3}+x^{2}+\left(1+u^{\mathfrak{t}}\right) x+1\right) .
\end{aligned}
$$

Example 3.8 Let $R_{k}=\mathbb{F}_{4}[u] /\left\langle u^{k}\right\rangle, m=7, u^{k}=0, \mathfrak{t} \geq\left\lceil\frac{k}{2}\right\rceil, \alpha=2$ be an even integer, implies $\alpha|(m-1)=2| 6, \operatorname{ord}_{7}(4)=\frac{m-1}{\alpha}=3$, then by Conjecture 3.5,

$$
\begin{aligned}
x^{7}-1 & =(x-1)\left(x^{3}+x+1\right)\left(x^{3}+x^{2}+1\right), \\
x^{7}-\left(1+u^{t}\right) & =\left(x+1+u^{t}\right)\left(x^{3}+x+1+u^{t}\right)\left(x^{3}+\left(1+u^{t}\right) x^{2}+1+u^{t}\right) .
\end{aligned}
$$

The proof of Theorem 3.9 is similar to that of Theorem 3.2, and is omitted.

Theorem 3.9 Assume that the factorization of $x^{m}-\lambda$ into basic irreducible polynomials over $R_{k}=\frac{\mathbb{F}_{q}[u]}{\left\langle u^{k}\right\rangle}$ is of the form of

1) case (1) in Conjecture 3.5, the total number of self-dual (resp. LCD) double $\lambda$-circulant codes over $R_{k}$ is

$$
B q^{\frac{(m-1)(k-1)}{2}}\left(q^{\frac{m-1}{2 \alpha}}+1\right)^{\alpha}\left(\operatorname{resp} \cdot(q-2) q^{m(k-1)}\left(q^{\frac{m-1}{\alpha}}-\left(q^{\frac{m-1}{2 \alpha}}+1\right)\right)^{\alpha}\right) .
$$


2) case (2) in Conjecture 3.5, the total number of self-dual (resp. LCD) double $\lambda$-circulant codes over $R_{k}$ is

$$
B q^{\frac{(m-1)(k-1)}{2}}\left(q^{\frac{m-1}{\alpha}}-1\right)^{\frac{\alpha}{2}}\left(\operatorname{resp} \cdot(q-2) q^{k-1}\left(q^{\frac{2 k(m-1)}{\alpha}}-q^{\frac{2(m-1)(k-1)}{\alpha}}\left(q^{\frac{m-1}{\alpha}}-1\right)\right)^{\frac{\alpha}{2}}\right) .
$$

\section{Main results}

Firstly, we give some lemmas as follows.

A. Case (1) in Lemma 3.4 In this case, by the Chinese Remainder Theorem (CRT), we have

$$
\begin{aligned}
\frac{R_{k}[x]}{\left\langle x^{m}+1\right\rangle} & \simeq \frac{R_{k}[x]}{\langle h(x)\rangle} \oplus \frac{R_{k}[x]}{\left\langle h^{*}(x)\right\rangle} \\
& \simeq \frac{\mathbb{F}_{q}[u, x]}{\left\langle u^{k}, h(x)\right\rangle} \oplus \frac{\mathbb{F}_{q}[u, x]}{\left\langle u^{k}, h^{*}(x)\right\rangle} \\
& \simeq\left(\mathbb{F}_{q^{n-1}}+u \mathbb{F}_{q^{n-1}}+\cdots+u^{k-1} \mathbb{F}_{q^{n-1}}\right) \oplus\left(\mathbb{F}_{q^{n-1}}+u \mathbb{F}_{q^{n-1}}+\cdots+u^{k-1} \mathbb{F}_{q^{n-1}}\right) \\
& \simeq R_{k\left(\frac{m}{2}\right)} \oplus R_{k\left(\frac{m}{2}\right)} .
\end{aligned}
$$

Lemma 4.1 If $0 \neq \varepsilon=(\mu, v) \in C$, and $C=\langle(1, h)\rangle$ is a double negacirculant code over $R_{k}$. Then

(1) there are at most $q^{\frac{m(2 k-1)}{2}}$ generators $(1, h)$ such that $\varepsilon=(\mu, v) \in C$.

(2) there are at most $q^{\frac{m(k-1)}{2}}$ generators $(1, h)$ such that $\varepsilon=(\mu, v) \in C$ and $C=C^{\perp}$.

(3) there are at most $q^{\frac{m(2 k-1)}{2}}$ generators $(1, h)$ such that $\varepsilon=(\mu, v) \in C$ and $C \cap$ $C^{\perp}=\{0\}$.

Proof By the CRT, $(\mu, v)=\left(\mu^{\prime}, v^{\prime}\right) \oplus\left(\mu^{\prime \prime}, v^{\prime \prime}\right)$. Since $(\mu, v) \in C$, then $v=\mu h$, $v^{\prime}=\mu^{\prime} h^{\prime}$ and $v^{\prime \prime}=\mu^{\prime \prime} h^{\prime \prime}$, where $\mu^{\prime}, v^{\prime}, h^{\prime} \in R_{k}[x] /\langle h(x)\rangle=R_{k\left(\frac{m}{2}\right)}$ and $\mu^{\prime \prime}, v^{\prime \prime}$, $h^{\prime \prime} \in R_{k}[x] /\left\langle h^{*}(x)\right\rangle=R_{k\left(\frac{m}{2}\right)}$. Let $h^{\prime}=h^{\prime(0)}+u h^{(1)}+\cdots+u^{k-1} h^{\prime(k-1)}$ and $h^{\prime \prime}=$ $h^{\prime \prime(0)}+u h^{\prime \prime(1)}+\cdots+u^{k-1} h^{\prime \prime(k-1)}$, where $h^{\prime(i)}, h^{\prime \prime(i)} \in \mathbb{F}_{q^{\frac{m}{2}}}, 0 \leq i \leq k-1$.

(1) In the first constituent of $C$, we discuss on the unit character of $\mu^{\prime}$ as follows.

- If $\mu^{\prime} \in R_{k\left(\frac{m}{2}\right)}^{\times}$, there exists only one solution $h^{\prime}=\frac{v^{\prime}}{\mu^{\prime}}$.

- If $\mu^{\prime} \in R_{k\left(\frac{m}{2}\right)} \backslash\left\{R_{k\left(\frac{m}{2}\right)}^{\times} \cup\{0\}\right\}$, then $\mu^{\prime}=u^{l} \mu^{\prime(l)}+u^{l+1} \mu^{\prime(l+1)}+\cdots+u^{k-1} \mu^{\prime(k-1)}$ where $1 \leq l \leq k-1, \mu^{\prime(l)} \in \mathbb{F}_{q^{\frac{m}{2}}}, \mu^{\prime(i)} \in \mathbb{F}_{q^{\frac{m}{2}}}, l+1 \leq i \leq k-1$ and $v^{\prime}=u^{l} v^{\prime(l)}+u^{l+1} v^{\prime(l+1)}+\cdots+u^{k-1} v^{\prime(k-1)}$ where $v^{\prime(j)} \in \mathbb{F}_{q^{\frac{m}{2}}}, l \leq j \leq k-1$. Since $v^{\prime}=\mu^{\prime} h^{\prime}, u^{k}=0$, then $v^{\prime}=u^{l} v^{(l)}+u^{l+1} v^{(l+1)}+\cdots+u^{k-1} v^{\prime(k-1)}=$ $\left(u^{l} \mu^{\prime(l)}+u^{l+1} \mu^{\prime(l+1)}+\cdots+u^{k-1} \mu^{\prime(k-1)}\right) h^{\prime}=u^{l} \mu^{\prime(l)} h^{\prime(0)}+u^{l+1}\left(\mu^{\prime(l+1)} h^{\prime(0)}+\right.$ $\left.\mu^{\prime(l)} h^{\prime(1)}\right)+\cdots+u^{k-1}\left(\mu^{\prime(k-1)} h^{\prime(0)}+\mu^{\prime(k-2)} h^{\prime(1)}+\cdots+\mu^{\prime(l)} h^{\prime(k-1-l)}\right)$. Hence, we have

$$
\left\{\begin{array}{l}
v^{\prime(l)}=\mu^{\prime(l)} h^{\prime(0)} \\
v^{\prime(l+1)}=\mu^{\prime(l+1)} h^{\prime(0)}+\mu^{\prime(l)} h^{\prime(1)} \\
\cdots \\
v^{\prime(k-1)}=\mu^{\prime(k-1)} h^{\prime(0)}+\mu^{\prime(k-2)} h^{\prime(1)}+\cdots+\mu^{\prime(l)} h^{\prime(k-1-l)}
\end{array}\right.
$$


By (7), we can get

$$
\left(\begin{array}{cccc}
\mu^{\prime(l)} & 0 & \cdots & 0 \\
\mu^{\prime(l+1)} & \mu^{\prime(l)} & \cdots & 0 \\
\vdots & \vdots & \vdots & \vdots \\
\mu^{\prime(k-1)} & \mu^{\prime(k-2)} & \cdots & \mu^{\prime(l)}
\end{array}\right) .
$$

Since $\mu^{\prime(l)} \in \mathbb{F}_{q^{\frac{m}{2}}}^{*}$, the determinant of the matrix (8) is not equal to 0 , then $h^{\prime(0)}, h^{\prime(1)}, \cdots, h^{\prime(k-1-l)}$ have a unique solution, $h^{\prime(k-l)}, h^{\prime(k-l+1)}, \cdots, h^{\prime(k-1)} \in$ $\mathbb{F}_{q^{\frac{m}{2}}}$. Thus there are at most $q^{\frac{m l}{2}}$ choices for $h^{\prime}$. When $l=k-1$, we can get the maximum possible for $h^{\prime}$, i.e., there are at most $q^{\frac{m(k-1)}{2}}$ choices for $h^{\prime}$.

- If $\mu^{\prime}=0$, then $h^{\prime} \in R_{k\left(\frac{m}{2}\right)}$, there are $q^{\frac{k m}{2}}$ choices for $h^{\prime}$.

Using the same argument as above in the second constituent of $C$, there are also at most $q^{\frac{k m}{2}}$ choices for $h^{\prime \prime}$. But $\varepsilon \neq 0$, then $\mu^{\prime}$ and $\mu^{\prime \prime}$ can not be zero simultaneously. Hence there are at most $q^{\frac{m(k-1)}{2}} \times q^{\frac{k m}{2}}$ generators $(1, h)$ such that $\varepsilon \in C$.

(2) Since $C$ is a self-dual double negacirculant code, then

$$
\left\langle\left(1, h^{\prime}\right),\left(1, h^{\prime \prime}\right)\right\rangle=1+h^{\prime} h^{\prime \prime}=0 .
$$

It is equivalent to

$$
\left\{\begin{array}{l}
h^{\prime(0)} h^{\prime \prime(0)}=-1, \\
h^{\prime(0)} h^{\prime \prime(1)}+h^{\prime(1)} h^{\prime \prime(0)}=0, \\
h^{\prime(0)} h^{\prime \prime(2)}+h^{\prime(1)} h^{\prime \prime(1)}+h^{\prime(2)} h^{\prime \prime(0)}=0, \\
\cdots \\
h^{\prime(0)} h^{\prime \prime(k-1)}+h^{\prime(1)} h^{\prime \prime(k-2)}+\cdots+h^{\prime(k-1)} h^{\prime \prime(0)}=0 .
\end{array}\right.
$$

Combining with the proof of (1), we have:

- if $\mu^{\prime}, \mu^{\prime \prime} \in R_{k\left(\frac{m}{2}\right)}^{\times}$, we know that $h^{\prime}=\frac{v^{\prime}}{\mu^{\prime}}$ and $h^{\prime \prime}=\frac{v^{\prime \prime}}{\mu^{\prime \prime}}$, then there are at most one generator $(1, h)$.

- if $\mu^{\prime} \in R_{k\left(\frac{m}{2}\right)}^{\times}, \mu^{\prime \prime} \in R_{k\left(\frac{m}{2}\right)} \backslash\left\{R_{k\left(\frac{m}{2}\right)}^{\times} \cup\{0\}\right\}$, we know that $h^{\prime}=\frac{v^{\prime}}{\mu^{\prime}}$ by (9), $h^{\prime \prime}$ can be uniquely fixed, then there are at most one generator $(1, h)$.

- if $\mu^{\prime} \in R_{k\left(\frac{m}{2}\right)}^{\times}, \mu^{\prime \prime}=0$, we know that $h^{\prime}=\frac{v^{\prime}}{\mu^{\prime}}$ and $h^{\prime \prime}$ is free, by (10), then there are at most one generator $(1, h)$.

- if $\mu^{\prime}, \mu^{\prime \prime} \in R_{k\left(\frac{m}{2}\right)} \backslash\left\{R_{k\left(\frac{m}{2}\right)}^{\times} \cup\{0\}\right\}$, we know that $h^{\prime(0)}$ can be uniquely fixed, $h^{\prime(1)}, h^{\prime(2)}, \cdots, h^{\prime(k-1)} \in \mathbb{F}_{q^{\frac{m}{2}}}, h^{\prime \prime(0)}$ can be uniquely fixed, $h^{\prime \prime(1)}, h^{\prime \prime(2)}, \cdots, h^{\prime \prime(k-1)} \in \mathbb{F}_{q^{\frac{m}{2}}}$, and because of (10), then there are at most $q^{\frac{m(k-1)}{2}}$ generators $(1, h)$.

- if $\mu^{\prime} \in R_{k\left(\frac{m}{2}\right)} \backslash\left\{R_{k\left(\frac{m}{2}\right)}^{\times} \cup\{0\}\right\}, \mu^{\prime \prime}=0$, we know that $h^{\prime(0)}$ can be uniquely fixed, $h^{\prime(1)}, h^{\prime(2)}, \cdots, h^{\prime(k-1)} \in \mathbb{F}_{q}{ }^{\frac{m}{2}}, h^{\prime \prime} \in R_{k\left(\frac{m}{2}\right)}$, and because of (10), then there are at most $q^{\frac{m(k-1)}{2}}$ generators $(1, h)$.

(3) Since $C$ is an LCD double negacirculant code, then

$$
\left\langle\left(1, h^{\prime}\right),\left(1, h^{\prime \prime}\right)\right\rangle=1+h^{\prime} h^{\prime \prime} \in R_{k\left(\frac{m}{2}\right)}^{\times} .
$$

Using the similar way, there are at most $q^{\frac{m(2 k-1)}{2}}$ generators $(1, h)$ such that $\varepsilon=$ $(\mu, \nu) \in C$ and $C \cap C^{\perp}=\{0\}$. We have thus proved the lemma. 
B. Case (2) in Lemma 3.4 In this case, by the CRT, we have

$$
\begin{aligned}
\frac{R_{k}[x]}{\left\langle x^{m}+1\right\rangle} & \simeq \bigoplus_{i=1}^{2}\left(\frac{R_{k}[x]}{\left\langle h_{i}(x)\right\rangle} \oplus \frac{R_{k}[x]}{\left\langle h_{i}^{*}(x)\right\rangle}\right) \\
& \simeq \bigoplus_{i=1}^{2}\left(\frac{\mathbb{F}_{q}[u, x]}{\left\langle u^{2}, h_{i}(x)\right\rangle} \oplus \frac{\mathbb{F}_{q}[u, x]}{\left\langle u^{2}, h_{i}^{*}(x)\right\rangle}\right) \\
& \simeq R_{k\left(\frac{m}{4}\right)} \oplus R_{k\left(\frac{m}{4}\right)} \oplus R_{k\left(\frac{m}{4}\right)} \oplus R_{k\left(\frac{m}{4}\right)}
\end{aligned}
$$

Lemma 4.2 If $C=\langle(1, h)\rangle$ is a double negacirculant code over $R_{k}$, and $0 \neq \varepsilon=(\mu, v) \in$ $C$, then

(1) there are at most $q^{\frac{m(4 k-1)}{4}}$ generators $(1, h)$ such that $\varepsilon=(\mu, v) \in C$.

(2) there are at most $q^{\frac{m(2 k-1)}{4}}$ generators $(1, h)$ such that $\varepsilon=(\mu, v) \in C$ and $C=C^{\perp}$.

(3) there are at most $q^{\frac{m(4 k-3)}{4}}\left(q^{\frac{m}{2}}-q^{\frac{m}{4}}+1\right)$ generators $(1, h)$ such that $\varepsilon=(\mu, v) \in C$ and $C \cap C^{\perp}=\{0\}$.

Proof By the CRT, $(\mu, v)=\bigoplus_{i=1}^{2}\left(\left(\mu_{i}^{\prime}, v_{i}^{\prime}\right) \oplus\left(\mu_{i}^{\prime \prime}, v_{i}^{\prime \prime}\right)\right)$. Since $(\mu, v) \in C$, then $v=\mu h$, $v_{i}^{\prime}=\mu_{i}^{\prime} h_{i}^{\prime}$ and $v_{i}^{\prime \prime}=\mu_{i}^{\prime \prime} h_{i}^{\prime \prime}$, where $\mu_{i}^{\prime}, v_{i}^{\prime}, h_{i}^{\prime} \in R_{k\left(\frac{m}{4}\right)}$ and $\mu_{i}^{\prime \prime}, v_{i}^{\prime \prime}, h_{i}^{\prime \prime} \in R_{k\left(\frac{m}{4}\right)}$. Let $h_{i}^{\prime}=h_{i}^{\prime(0)}+u h_{i}^{\prime(1)}+\cdots+u^{k-1} h_{i}^{\prime(k-1)}$ and $h_{i}^{\prime \prime}=h_{i}^{\prime \prime(0)}+u h_{i}^{\prime \prime(1)}+\cdots+u^{k-1} h_{i}^{\prime \prime(k-1)}$, where $h_{i}^{\prime(j)}, h_{i}^{\prime \prime(j)} \in \mathbb{F}_{q^{\frac{m}{4}}}, 1 \leq i \leq 2,0 \leq j \leq k-1$.

(1) In the first constituent of $C$, we discuss on the unit character of $\mu_{1}^{\prime}$ as follows.

- If $\mu_{1}^{\prime} \in R_{k\left(\frac{m}{4}\right)}^{\times}$, there exists only one solution $h_{1}^{\prime}=\frac{v_{1}^{\prime}}{\mu_{1}^{\prime}}$.

- If $\mu_{1}^{\prime} \in R_{k\left(\frac{m}{4}\right)} \backslash\left\{R_{k\left(\frac{m}{4}\right)}^{\times} \cup\{0\}\right\}$, then $\mu_{1}^{\prime}=u^{l} \mu_{1}^{\prime(l)}+u^{l+1} \mu_{1}^{\prime(l+1)}+\cdots+$ $u^{k-1} \mu_{1}^{\prime(k-1)}, 1 \leq l \leq k-1, \mu_{1}^{\prime(l)} \in \mathbb{F}_{q^{\frac{m}{4}}}, \mu_{1}^{\prime(i)} \in \mathbb{F}_{q^{\frac{m}{4}}}, l+1 \leq i \leq k-1$ and $v_{1}^{\prime}=u^{l} v_{1}^{\prime(l)}+u^{l+1} v_{1}^{(l)}+\cdots+u^{k-1} v_{1}^{\prime(k-1)}, v_{1}^{\prime(j)} \in \mathbb{F}_{q^{\frac{m}{4}}}, l \leq i \leq k-1$. Since $v_{1}^{\prime}=\mu_{1}^{\prime} h_{1}^{\prime}, u^{k}=0$, then $v_{1}^{\prime}=u^{l} v_{1}^{\prime(l)}+u^{l+1} v_{1}^{\prime(l)}+\cdots+u^{k-1} v_{1}^{\prime(k-1)}=$ $\left(u^{l} \mu_{1}^{\prime(l)}+u^{l+1} \mu_{1}^{\prime(l+1)}+\cdots+u^{k-1} \mu_{1}^{\prime(k-1)}\right) h_{1}^{\prime}=u^{l} \mu_{1}^{\prime(l)} h_{1}^{\prime(0)}+u^{l+1}\left(\mu_{1}^{\prime(l+1)} h_{1}^{\prime(0)}+\right.$ $\left.\mu_{1}^{\prime(l)} h_{1}^{\prime(1)}\right)+\cdots+u^{k-1}\left(\mu_{1}^{\prime(k-1)} h_{1}^{\prime(0)}+\mu_{1}^{\prime(k-2)} h_{1}^{\prime(1)}+\cdots+\mu_{1}^{\prime(l)} h_{1}^{\prime(k-1-l)}\right)$. Hence, we obtain

$$
\left\{\begin{array}{l}
v_{1}^{\prime(l)}=\mu_{1}^{\prime(l)} h_{1}^{\prime(0)} \\
v_{1}^{\prime(l+1)}=\mu_{1}^{\prime(l+1)} h_{1}^{\prime(0)}+\mu_{1}^{\prime(l)} h_{1}^{\prime(1)} \\
\cdots \\
v_{1}^{\prime(k-1)}=\mu_{1}^{\prime(k-1)} h_{1}^{\prime(0)}+\mu_{1}^{\prime(k-2)} h_{1}^{\prime(1)}+\cdots+\mu_{1}^{\prime(l)} h_{1}^{\prime(k-1-l)}
\end{array}\right.
$$

By (12), we can get

$$
\left(\begin{array}{cccc}
\mu_{1}^{\prime(l)} & 0 & \cdots & 0 \\
\mu_{1}^{\prime(l+1)} & \mu_{1}^{\prime(l)} & \cdots & 0 \\
\vdots & \vdots & \vdots & \vdots \\
\mu_{1}^{\prime(k-1)} & \mu_{1}^{\prime(k-2)} & \cdots & \mu_{1}^{\prime(l)}
\end{array}\right)
$$


Since $\mu_{1}^{\prime(l)} \in \mathbb{F}_{q^{\frac{m}{4}}}$, the determinant of the matrix (13) is not equal to 0 , then $h_{1}^{\prime(0)}, h_{1}^{\prime(1)}, \cdots, h_{1}^{\prime(k-1-l)}$ have a unique solution, $h_{1}^{\prime(k-l)}, h_{1}^{\prime(k-l+1)}, \cdots, h_{1}^{\prime(k-1)} \in$ $\mathbb{F}_{q^{\frac{m}{4}}}$. Thus, there are at most $q^{\frac{m l}{4}}$ choices for $h_{1}^{\prime}$. When $l=k-1$, we can get the maximum possible for $h_{1}^{\prime}$, i.e., there are at most $q^{\frac{m(k-1)}{4}}$ choices for $h_{1}^{\prime}$.

- If $\mu_{1}^{\prime}=0$, then $h_{1}^{\prime} \in R_{k\left(\frac{m}{4}\right)}$, there are $q^{\frac{k m}{4}}$ choices for $h_{1}^{\prime}$.

Using the same argument as above in the other constituent of $C$, there are also at most $q^{\frac{k m}{4}}$ choices for $h_{2}^{\prime}, h_{1}^{\prime \prime}, h_{2}^{\prime \prime}$. But $\varepsilon \neq 0$, then $\mu_{1}^{\prime}, \mu_{2}^{\prime}, \mu_{1}^{\prime \prime}$ and $\mu_{2}^{\prime \prime}$ can not be zero simultaneously. Hence, there are at most $q^{\frac{m(k-1)}{4}} \times\left(q^{\frac{k m}{4}}\right)^{3}$ generators $(1, h)$ such that $\varepsilon \in C$.

(2) Since $C$ is a self-dual double negacirculant code, then

$$
\left\{\begin{array}{l}
\left\langle\left(1, h_{1}^{\prime}\right),\left(1, h_{1}^{\prime \prime}\right)\right\rangle=0 \\
\left\langle\left(1, h_{2}^{\prime}\right),\left(1, h_{2}^{\prime \prime}\right)\right\rangle=0
\end{array}\right.
$$

Combining with the proof of (1), similar to the discussion of (2) in Lemma 4.1, there are at most $q^{\frac{(2 k-1) m}{4}}$ generators $(1, h)$ such that $\varepsilon=(\mu, v) \in C$ and $C=C^{\perp}$.

(3) Since $C$ is an LCD double negacirculant code, then

$$
\left\langle\left(1, h_{i}^{\prime}\right),\left(1, h_{i}^{\prime \prime}\right)\right\rangle=1+h_{i}^{\prime} h_{i}^{\prime \prime} \in R_{k\left(\frac{m}{4}\right)}^{\times}, i=1,2 .
$$

Using the similar way, there are at most $q^{\frac{m(4 k-3)}{4}}\left(q^{\frac{m}{2}}-q^{\frac{m}{4}}+1\right)$ generators $(1, h)$ such that $\varepsilon=(\mu, v) \in C$ and $C \cap C^{\perp}=\{0\}$. We have thus proved the lemma.

\section{Case (1) in Theorem 3.9}

Lemma 4.3 If $0 \neq \varepsilon=(\mu, v) \in C$, and if there exists a positive integer $i$ such that $\mu$ is not generated by $g_{i}(x)$, and if, furthermore, $C=\langle(1, h)\rangle$ is a double $\lambda$-circulant code over $R_{k}$, then

(1) there are at most $q^{\frac{m(k \alpha-1)+1}{\alpha}}$ generators $(1, h)$ such that $\varepsilon \in C$.

(2) there are at most $B q^{\frac{(m-1)(k-1)}{2}}\left(q^{\frac{m-1}{2 \alpha}}+1\right)^{\alpha-1}$ generators $(1, h)$ such that $\varepsilon \in C$ and $C=C^{\perp}$.

(3) there are at most $(q-2) q^{m(k-1)}\left(q^{\frac{m-1}{\alpha}}-q^{\frac{m-1}{2 \alpha}}-1\right)^{\alpha-1}$ generators $(1, h)$ such that $\varepsilon=(\mu, v) \in C$ and $C \cap C^{\perp}=\{0\}$.

Proof By the CRT, we have $(\mu, \nu) \simeq\left(\mu_{0}, v_{0}\right) \oplus\left(\bigoplus_{i=1}^{\alpha}\left(\mu_{i}, v_{i}\right)\right)$. Since $\varepsilon=(\mu, v) \in C$, then $v=\mu h, v_{0}=\mu_{0} h_{0}$ and $v_{i}=\mu_{i} h_{i}$, where $\mu_{0}, v_{0}, h_{0} \in R_{k}=R_{k}[x] /\langle A(x)\rangle$ and $\mu_{i}$, $v_{i}, h_{i} \in R_{k\left(\frac{m-1}{\alpha}\right)}=R_{k}[x] /\left\langle g_{i}(x)\right\rangle$. Let $h_{0}=h_{0}^{(0)}+u h_{0}^{(1)}+\cdots+u^{k-1} h_{0}^{(k-1)}$ and $h_{i}=$ $h_{i}^{(0)}+u h_{i}^{(1)}+\cdots+u^{k-1} h_{i}^{(k-1)}$, where $h_{0}^{(j)} \in \mathbb{F}_{q}, h_{i}^{(j)} \in \mathbb{F}_{q^{\frac{m-1}{\alpha}}}, 1 \leq i \leq \alpha, 0 \leq j \leq k-1$.

(1) In the first constituent of $C$, we discuss on the unit character of $\mu_{0}$ as follows.

- If $\mu_{0} \in R_{k}^{\times}$, there exists unique solution $h_{0}=\frac{v_{0}}{\mu_{0}}$.

- If $\mu_{0} \in R_{k} \backslash\left\{R_{k}^{\times} \cup\{0\}\right\}$, similar to discuss in Lemma 4.1, there are at most $q^{k-1}$ choices for $h_{0}$.

- If $\mu_{0}=0$, then $h_{0}$ is arbitrary in $R_{k}$, there are $q^{k}$ choices for $h_{0}$. 
In the $i$ th constituent of $C$, we discuss on the unit character of $\mu_{i}$ as follows.

- If $\mu_{i} \in R_{k\left(\frac{m-1}{\alpha}\right)}^{\times}$, there exists only one solution $h_{i}=\frac{\nu_{i}}{\mu_{i}}$.

- If $\mu_{i} \in R_{k\left(\frac{m-1}{\alpha}\right)} \backslash\left\{R_{k\left(\frac{m-1}{\alpha}\right)}^{\times} \cup\{0\}\right\}$, this case can be discussed similarly. Hence, there are at most $q^{\frac{(m-1)(k-1)}{\alpha}}$ choices for $h_{i}$.

- If $\mu_{i}=0$, there are $q^{\frac{k(m-1)}{\alpha}}$ choices for $h_{1}^{\prime}$.

Thus there are at most $q^{\frac{m(k \alpha-1)+1}{\alpha}}$ generators $(1, h)$ such that $\varepsilon \in C$.

(2) In the first constituent of $C$, there are at most $B$ generators $\left(1, h_{0}\right)$ such that $C_{0}$ is a self-dual double $\lambda$-circulant code over $R_{k}$ by Theorem 3.2.

In the $i$ th constituent of $C$, combining with (1), we can get

- if $\mu_{i} \in R_{k\left(\frac{m-1}{\alpha}\right)}^{\times}$, there exists only one solution $h_{i}=\frac{\nu_{i}}{\mu_{i}}$.

- if $\mu_{i} \in R_{k\left(\frac{m-1}{\alpha}\right)} \backslash\left\{R_{k\left(\frac{m-1}{\alpha}\right)}^{\times} \cup\{0\}\right\}, \quad h_{i}^{(0)}$ can be uniquely fixed, $h_{i}^{(1)}, h_{i}^{(2)} \cdots, h_{i}^{(k-1)} \in \mathbb{F}_{q^{\frac{m-1}{\alpha}}}$. And because $C$ is a self-dual double $\lambda$-circulant code, then $\left\langle\left(1, h_{i}\right) \cdot\left(1, h_{i}\right)\right\rangle_{H}=1+h_{i} \overline{h_{i}}=0$, which implies

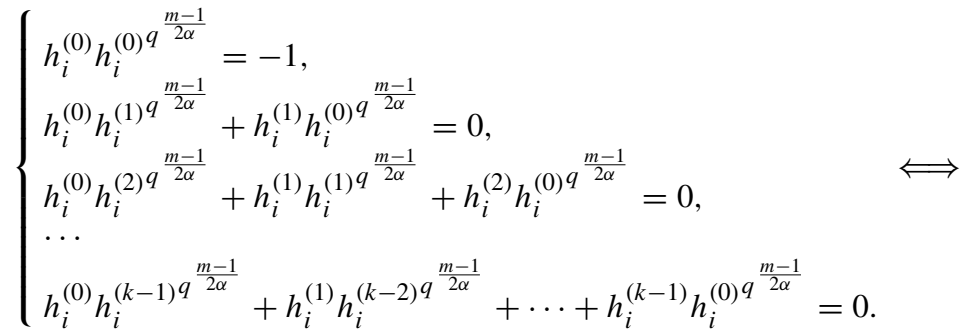

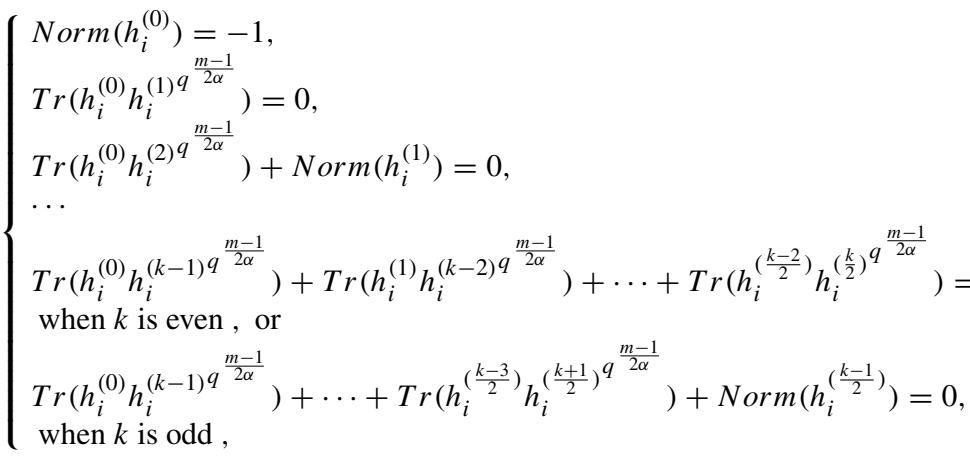

then there are at most $q^{\frac{(m-1)(k-1)}{2 \alpha}}$ choices for $h_{i}$.

- if $\mu_{i}=0$, since $C$ is a self-dual double $\lambda$-circulant code, there are at most $q^{\frac{(m-1)(k-1)}{2 \alpha}}\left(q^{\frac{m-1}{2 \alpha}}+1\right)$ choices for $h_{i}$.

Thus there are at most $B q^{\frac{(m-1)(k-1)}{2}}\left(q^{\frac{m-1}{2 \alpha}}+1\right)^{\alpha-1}$ generators $(1, h)$ such that $\varepsilon \in C$ and $C=C^{\perp}$.

(3) Since $C$ is an LCD double $\lambda$-circulant code, then

$$
\left\langle\left(1, h_{i}\right),\left(1, h_{i}\right)\right\rangle_{H}=1+h_{i} \bar{h}_{i} \in R_{k}^{\times}, i=1,2, \cdots, \alpha .
$$


Hence, there are at most $(q-2) q^{m(k-1)}\left(q^{\frac{m-1}{\alpha}}-q^{\frac{m-1}{2 \alpha}}-1\right)^{\alpha-1}$ generators $(1, h)$ such that $\varepsilon=(\mu, v) \in C$ and $C \cap C^{\perp}=\{0\}$.

\section{Case (2) in Theorem 3.9}

Lemma 4.4 If $C=\langle(1, h)\rangle$ is a double $\lambda$-circulant code over $R_{k}$, such that $0 \neq \varepsilon=$ $(\mu, v) \in C$, and that there exists a positive integer $i$ such that $\mu$ is not generated by $h_{i}(x)$, then

(1) there are at most $q^{\frac{m(k \alpha-1)+1}{\alpha}}$ generators $(1, h)$ such that $\varepsilon \in C$.

(2) there are at most $B q^{\frac{(m-1)(k \alpha-2)}{2 \alpha}}$ generators $(1, h)$ such that $\varepsilon \in C$ and $C=C^{\perp}$.

(3) there are at most $(q-2) q^{\frac{(m-1)(\alpha k-\alpha+1)}{\alpha}}\left(q^{\frac{2(m-1)}{\alpha}}-q^{\frac{m-1}{\alpha}}+1\right)^{\frac{\alpha}{2}-1}$ generators $(1, h)$ such that $\varepsilon=(\mu, v) \in C$ and $C \cap C^{\perp}=\{0\}$.

Proof Again using the CRT, we have $(\mu, v) \simeq\left(\mu_{0}, v_{0}\right) \oplus\left(\bigoplus_{j=1}^{\alpha / 2}\left(\left(\mu_{j}^{\prime}, v_{j}^{\prime}\right) \oplus\left(\mu_{j}^{\prime \prime}, v_{j}^{\prime \prime}\right)\right)\right)$. Since $\varepsilon=(\mu, v) \in C$, then $v=\mu h, v_{0}=\mu_{0} h_{0}, v_{j}^{\prime}=\mu_{j}^{\prime} h_{j}^{\prime}$ and $v_{j}^{\prime \prime}=\mu_{j}^{\prime \prime} h_{j}^{\prime \prime}$, where $\mu_{0}$, $v_{0}, h_{0} \in R_{k}=R_{k}[x] /\langle A(x)\rangle, \mu_{j}^{\prime}, v_{j}^{\prime}, h_{j}^{\prime} \in R_{k\left(\frac{m-1}{\alpha}\right)}=R_{k}[x] /\left\langle h_{j}(x)\right\rangle$ and $\mu_{j}^{\prime \prime}, v_{j}^{\prime \prime}, h_{j}^{\prime \prime} \in$ $R_{k\left(\frac{m-1}{\alpha}\right)}=R_{k}[x] /\left\langle h_{j}^{*}(x)\right\rangle$. Let $h_{0}=h_{0}^{(0)}+u h_{0}^{(1)}+\cdots+u^{k-1} h_{0}^{(k-1)}, h_{j}^{\prime}=h_{j}^{(0)}+u h_{j}^{(1)}+$ $\cdots+u^{k-1} h_{j}^{\prime(k-1)}, h_{j}^{\prime \prime}=h_{j}^{\prime \prime(0)}+u h_{j}^{\prime \prime(1)}+\cdots+u^{k-1} h_{j}^{\prime \prime(k-1)}$, where $h_{0}^{(0)}, h_{0}^{(1)}, \cdots, h_{0}^{(k-1)} \in$ $\mathbb{F}_{q}, h_{j}^{\prime(0)}, h_{j}^{\prime(1)}, \cdots, h_{j}^{\prime(k-1)} \mathbb{F}_{q^{\frac{m-1}{\alpha}}}, h_{j}^{\prime \prime(0)}, h_{j}^{\prime \prime(1)}, \cdots, h_{j}^{\prime \prime(k-1)} \in \mathbb{F}_{q^{\frac{m-1}{\alpha}}}$.

(1) Since $0 \neq \varepsilon \in C$, there are at most $q^{\frac{m(k \alpha-1)+1}{\alpha}}$ generators $(1, h)$.

(2) In the first constituent of $C$, there are at most $B$ generators $\left(1, h_{0}\right)$ such that $C_{0}$ is a self-dual double $\lambda$-circulant code over $R_{k}$ according to Theorem 3.2.

In the $j$ th constituent of $C$, we have a similar discussion for pairs $\left(\mu_{j}^{\prime}, \mu_{j}^{\prime \prime}\right)$, there are at most $B q^{\frac{(m-1)(k \alpha-2)}{2 \alpha}}$ generators $(1, h)$ such that $\varepsilon \in C$ and $C=C^{\perp}$.

(3) Since $C$ is an LCD double $\lambda$-circulant code, then

$$
\left\langle\left(1, h_{j}^{\prime}\right),\left(1, h_{j}^{\prime \prime}\right)\right\rangle=1+h_{j}^{\prime} h_{j}^{\prime \prime} \in R_{k\left(\frac{m-1}{\alpha}\right)}^{\times}, j=1,2, \cdots, \frac{\alpha}{2} .
$$

Hence, there are at most $(q-2) q^{\frac{(m-1)(\alpha k-\alpha+1)}{\alpha}}\left(q^{\frac{2(m-1)}{\alpha}}-q^{\frac{m-1}{\alpha}}+1\right)^{\frac{\alpha}{2}-1}$ generators $(1, h)$ such that $\varepsilon=(\mu, v) \in C$ and $C \cap C^{\perp}=\{0\}$.

We are now ready for the main result of this paper.

Theorem 4.5 If $q$ is a power of prime, then there are infinite families of:

(1) self-dual (resp. LCD) negacirculant codes of index 2 over $R_{k}$ of relative distance $\delta$ satisfying $H_{q}(\delta) \geq \frac{1}{4 p^{k-1}}\left(\right.$ resp. $\left.H_{q}(\delta) \geq \frac{1}{4 p^{k-1}}\right)$ for case (1) in Lemma 3.4;

(2) self-dual (resp. LCD) negacirculant codes of index 2 over $R_{k}$ of relative distance $\delta$ satisfying $H_{q}(\delta) \geq \frac{1}{8 p^{k-1}}\left(\right.$ resp. $\left.H_{q}(\delta) \geq \frac{1}{8 p^{k-1}}\right)$ for case (2) in Lemma 3.4;

(3) self-dual (resp. LCD) $\lambda$-circulant codes of index 2 over $R_{k}$ of relative distance $\delta$ satisfying $H_{q}(\delta) \geq \frac{1}{4 \alpha p^{k-1}}\left(\right.$ resp. $\left.H_{q}(\delta) \geq \frac{1}{2 \alpha p^{k-1}}\right)$ for case $(1)$ in Theorem 3.9;

(4) self-dual (resp. LCD) $\lambda$-circulant codes of index 2 over $R_{k}$ of relative distance $\delta$ satisfying $H_{q}(\delta) \geq \frac{1}{2 \alpha p^{k-1}}\left(\right.$ resp. $\left.H_{q}(\delta) \geq \frac{1}{2 \alpha p^{k-1}}\right)$ for case $(2)$ in Theorem 3.9. 
Proof By the Gray map over $R_{k}$, we see that the Gray image of the several families of codes of length $2 m$ are linear codes of length $2 m p^{k-1}$. Combining Lemmas 3.4, 4.1, 4.2, 4.3, 4.4 and Theorem 3.9, the result follows by the same method as Theorem 5.2 in [20], so we omit the detailed proof here.

\section{Conclusion}

In the present paper, we have studied self-dual (resp. LCD) double $\lambda$-circulant codes over the ring $R_{k}=\mathbb{F}_{p}[u] /\left\langle u^{k}\right\rangle$,i.e., index 2 quasi-twisted codes with twisting constant $\lambda= \pm 1$ and $\lambda=1+w u^{\mathrm{t}}$.

We not only have considered the special factorization of $x^{m}+1$ to construct the double negacirculant codes when it factors into two (resp. four) basic irreducible factors reciprocal of each other for $m$ a power of 2 in [1,2], but also have studied another special kind of factorization, for $m$ odd prime, and $(m, q)=1$ when $x^{m}-\lambda$ factors into $\alpha+1$ basic irreducible polynomials with $\alpha \mid(m-1)$ and $\operatorname{ord}_{m}(q)=\frac{m-1}{\alpha}$. With this particular factorization, we have constructed self-dual (resp. LCD) quasi-twisted codes of index 2 over $R_{k}$, and derived an exact enumeration formula for this family of codes. Further, we have derived a modified Varshamov-Gilbert bound on the relative distance of the codes considered, building on exact enumeration results.

The main open problem is Conjecture 3.5. More general directions are quasi-twisted codes of index $>2$ and replacing $R_{k}$ by a general chain ring.

Publisher's Note Springer Nature remains neutral with regard to jurisdictional claims in published maps and institutional affiliations.

\section{References}

1. Alahmadi, A., Güneri, C., Özkaya, B., Shoaib, H., Solé, P.: On self-dual double negacirculant codes. Discret. Appl. Math. 222, 205-212 (2017)

2. Alahmadi, A., Güneri, C., Özkaya, B., Shoaib, H., Solé, P.: On linear complementary-dual multinegacirculant codes, Cryptography and Communications (2017)

3. Alahmadi, A., Özdemir, F., Solé, P.: On self-dual double circulant codes, Designs, Codes and Cryptography. https://doi.org/10.1007/s10623-017-0393-x (2017)

4. Carlet, C., Güneri, C., Özbudak, F., Solé, P.: A new concatenated type construction for LCD codes and isometry codes. Discret. Math. 341(3), 830-835 (2018)

5. Carlet, C., Mesnager, S., Tang, C.M., Qi, Y.F., Pellikaan, R.: Linear Codes Over $\mathbb{F}_{q}$ Are Equivalent to LCD Codes for $q>3$. IEEE Trans. Inf. Theory 64(4), 3010-3017 (2018)

6. Carlet, C., Guilley, S.: Complementary dual codes for counter-measures to side-channel attacks. Adv. Math. Commun. 10, 131-150 (2016)

7. Carlet, C., Guilley, S.: Statistical properties of side-channel and fault injection attacks using coding theory. Cryptogr. Commun. 10, 909-933 (2018)

8. Güneri, C., Özkaya, B., Solé, P.: Quasi-cyclic complementary dual codes. Finite Fields Appl. 42, 67-80 (2016)

9. Hooley, C.: On Artin's conjecture. J. Fur Die Reine Und Angewandte Mathematik 225, 209-220 (1967)

10. Huffman, W.C., Pless, V.: Fundamentals of error correcting codes. Cambridge University Press, Cambridge (2003)

11. Jia, Y.: On quasi-twisted codes over finite fields. Finite Fields Appl. 18, 237-257 (2012)

12. Kaya, A., Yildiz, B., Pasa, A.: New extremal binary self-dual codes from a modified four circulant construction. Discret. Math. 339, 1086-1094 (2016)

13. Ling, S., Solé, P.: On the algebraic structure of quasi-cyclic codes I: finite fields. IEEE Trans. Inf. Theory 47, 2751-2760 (2001) 
14. Ling, S., Solé, P.: On the algebraic structure of quasi-cyclic codes II: Chain rings, Des. Codes Crypt. 30(1), 113-130 (2003)

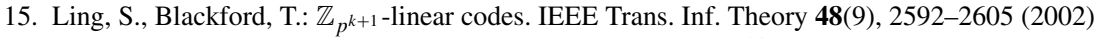

16. Moree, P.: Artin's primitive root conjecture a survey. Integers 10(6), 1305-1416 (2012)

17. Meyn, H.: Factorization of the cyclotomic polynomial $x^{2^{n}}+1$ over finite fields. Finite Fields Their Appl 2, 439-442 (1996)

18. Massey, J.L.: Linear codes with complementary duals. Discret. Math. 106(/107), 337-342 (1992)

19. Saleh, A., Esmaeili, M.: Some classes of quasi-twisted codes over finite chain rings, Journal of Applied Mathematics and Computing. https://doi.org/10.1007/s12190-017-1125-0 (2017)

20. Shi, M.J., Qian, L.Q., Solé, P.: On self-dual negacirculant codes of index two and four, Designs, Codes and Cryptography. https://doi.org/10.1007/s10623-017-0455-0 (2018)

21. Shi, M.J., Liu, Y., Solé, P.: Optimal two-weight codes from trace codes over $\mathbb{F}_{2}+u \mathbb{F}_{2}$. IEEE Commun. Lett. 20(12), 2346-2349 (2016)

22. Shi, M.J., Zhu, S.X., Yang, S.L.: A class of optimal $p$-ary codes from one-weight codes over $\mathbb{F}_{p}[u] /\left(u^{m}\right)$. J. Franklin Inst. 350(5), 929-937 (2013)

23. Shi, M.J., Wu, R.S., Liu, Y., Solé, P.: Two and three weight codes over $\mathbb{F}_{p}+u \mathbb{F}_{p}$. Cryptogr. Commun. 9, 637-646 (2017) 